\title{
A Secure Scheme for Wireless Sensor Networks Based on Grids and A bilinear Map
}

\author{
Yuquan Zhang ${ }^{1,2, a}$; Lei Wei ${ }^{3, b}$ \\ ${ }^{1}$ Shandong Women University, China \\ ${ }^{2}$ Wireless Sensing Institute, College of Information Science and Engineering, Qilu Normal University, \\ China \\ ${ }^{3}$ College of Physics and Electronic Engineering, Qilu Normal University, China \\ aemail:zyczyq@126.com; bemail:weilei76@126.com
}

Keywords: A bilinear map; security; grids.

Abstract: A WSNs(wireless sensor networks) secure strategy is presented by dividing sensing square into grids and utilizing a bilinear map methodology in this paper. Both ordinary sensor nodes and heterogeneous sensor nodes are dispersed in whole sensing area. All those sensor nodes establish their communication keys by employing a bilinear map. Analysis shows this scheme improves the WSN security, and has good network connectivity.

\section{Introduction}

Nowadays, a sensor node, a small and autonomous wireless device, has been facilitated to develop by the development of micro-electro-mechanical system (MEMS). Sensors have multiple abilities, such as sensing, calculating, and communication ${ }^{[1]}$.

Wireless sensor networks consist of numerous sensor nodes linked by a wireless transmission medium. The unique characters of sensor nodes are low wireless communication capacity, limited energy, limited storage memory and low computational ability.

Due to the hardware resource limitations, the wireless medium of WSN, and, in some cases, the hostile environments in which WSNs are deployed, wireless sensor networks are vulnerable to various malicious attacks ${ }^{[2]}$ including eavesdropping, masquerading, traffic-analysis, node capture, and so on.

Assuring the security for WSN is a challenging issue. The key management that thwarts the activities of malicious sensor nodes through generating secret keys in a secure way is an efficient method to guarantee wireless sensor networks secure.

L. Eschenauer and V. D. Gligor ${ }^{[3]}$ proposed a probabilistic key pre-distribution scheme, which is a basic strategy in many papers, to establish pairwise keys between two nodes. In this scheme, communication keys are set up through three steps: key predistribution, shared-key discovery, and path-key establishment. A big key pool is created and some keys are drawn out of the pool and stored into sensor memory in the first step. All sensors find their neighbors with their shared keys in their wireless communication ranges in the second step. Those sensors that share no common key are connected by two or more links in the last step. It has poor security because many keys are revealed, if several sensor nodes are captured. As a result, the communications are affected and the connectivity among sensors deteriorates. The strategy has further been improved by a $q$-composite key pre-distribution scheme a random pairwise key scheme ${ }^{[4]}$. The $q$-composite key pre-distribution also uses a key pool but requires two nodes compute a pairwise key from at least $q$ pre-distributed keys that they share. This strategy can improve the system security, but the connectivity declines. Liu D and $\mathrm{Ning}^{[5]}$ presented a general framework for polynomial pool-based pairwise key predistribution in sensor networks and two possible instantiations key predistribution schemes: random subset assignment and grid-based schemes. This strategy is secure and resists collusion. The disadvantage is that it will be more vulnerable to be compromised when the sensor network becomes larger.

In the paper, we give a key management scheme based on a bilinear map and the elliptic curve discrete logarithm for the wireless sensor networks security. The sensing area is divided into many 
different grids. Analysis demonstrates that this scheme enhances the resilience of WSNs, and has good network connectivity.

The rest of this paper is organized as follows. In section two, the method to divide sensing square into grids and distribute sensors is presented. Section three gives the key systems based on identity. Performance analysis for WSNs is given in the section three. Setting up pairwise key based a bilinear map is in the section four. Section five gives the security and connectivity analysis. The conclusion of this paper is in section six.

\section{Dividing sensing square and distributing sensors}

The sensing area is a square expressed as $S$ and both ordinary sensor nodes and heterogeneous sensor nodes are equally dispensed in $S$ in this scheme. The sensing square in the WSNs is divided into $8 \times n$ sections. In the Fig. 1, there are many concentric squares and the side length of the minimum square is $r$, the side length of the secondary minimum square is $2 r$, $\mathrm{L}$, the side length of the largest square is $n r$. All those concentric squares are divided into 8 sections equally. The section $\mathrm{OM}_{00} \mathrm{M}_{10}$ is expressed as $(0,0)$, the sector $\mathrm{OM}_{10} \mathrm{M}_{20}$ is expressed as $(0,1)$, $\mathrm{L}$, the sector $\mathrm{OM}_{70} \mathrm{M}_{00}$ is expressed as $(0,7)$, the section $M_{00} M_{01} M_{11} M_{10}$ is expressed as $(1,0)$, the section $M_{10} M_{11} M_{21} M_{20}$ is expressed as $(1,1), \mathrm{L}$, the section $\mathrm{M}_{70} \mathrm{M}_{71} \mathrm{M}_{01} \mathrm{M}_{00}$ is expressed as $(1,7)$, $\mathrm{L}$, the section $\mathrm{M}_{7(n-2)} \mathrm{M}_{7(n-1)} \mathrm{M}_{0(n-2)} \mathrm{M}_{0(n-1)}$ is expressed as $(n-1,7)$. Generally, sections are expressed as $(p, q)$.

We make an assumption that the area of the sector $\mathrm{OM}_{00} \mathrm{M}_{10}$ is $S_{0,0}$. The area $S_{1,0}$ of the section

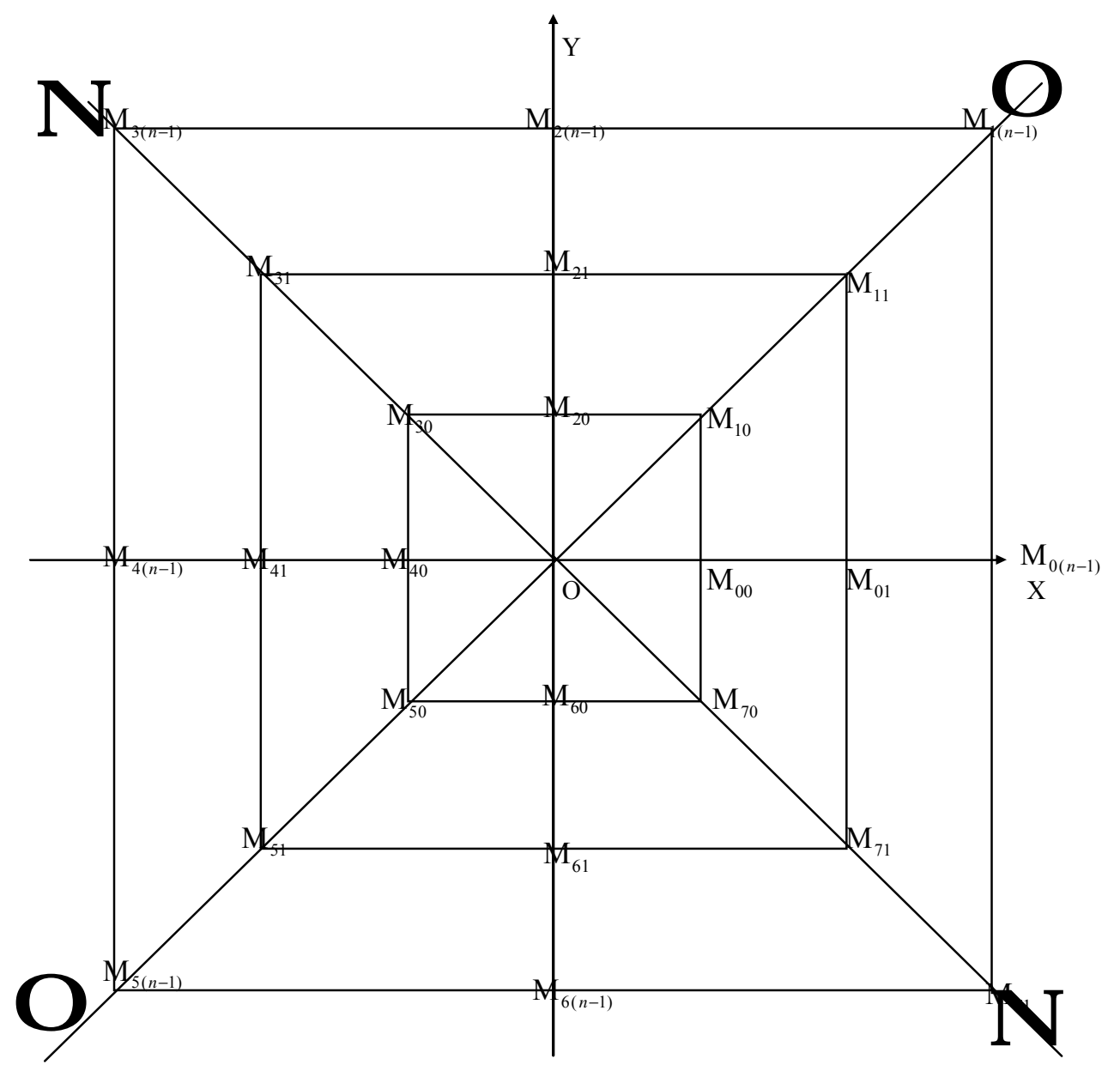

Fig. 1 The sensing square 
$\mathrm{M}_{00} \mathrm{M}_{01} \mathrm{M}_{11} \mathrm{M}_{10}$ is $3 S_{0,0}$, the area $S_{2,0}$ of the section $\mathrm{M}_{01} \mathrm{M}_{02} \mathrm{M}_{12} \mathrm{M}_{11}$ is $5 S_{0,0}$, $\mathrm{L}$, the area $S_{(n-1), 0}$ of the section $\mathrm{M}_{0(n-2)} \mathrm{M}_{0(n-1)} \mathrm{M}_{1(n-1)} \mathrm{M}_{1(n-2)}$ is $(2 n-1) S_{0,0}$. Samely, we calculate those areas of other sections.

We make an assumption that $\alpha$ ordinary nodes are dispensed in section $\mathrm{OM}_{00} \mathrm{M}_{10}$. Therefore, $3 \alpha$ ordinary sensors are dispensed in $\mathrm{M}_{00} \mathrm{M}_{01} \mathrm{M}_{11} \mathrm{M}_{10}, 5 \alpha$ ordinary sensors are dispensed in $\mathrm{M}_{01} \mathrm{M}_{02} \mathrm{M}_{12} \mathrm{M}_{11}, \mathrm{~L},(2 n-1) \alpha$ sensors are dispensed in $\mathrm{M}_{0(n-2)} \mathrm{M}_{0(n-1)} \mathrm{M}_{1(n-1)} \mathrm{M}_{1(n-2)}$. We make another assumption that heterogeneous sensors that have more storage memory, battery energy, communication capacity, and higher computational ability than ordinary sensors are dispensed in sensing area equally and $\beta$ heterogeneous sensors are dispensed in $\mathrm{OM}_{00} \mathrm{M}_{10}$. Then, $3 \beta$ heterogeneous sensors are dispensed in $\mathrm{M}_{00} \mathrm{M}_{01} \mathrm{M}_{11} \mathrm{M}_{10}$, $5 \beta$ heterogeneous sensors are dispensed in $\mathrm{M}_{01} \mathrm{M}_{02} \mathrm{M}_{12} \mathrm{M}_{11}$,,$(2 n-1) \beta$ heterogeneous sensors are dispensed in $\mathrm{M}_{0(n-2)} \mathrm{M}_{0(n-1)} \mathrm{M}_{1(n-1)} \mathrm{M}_{1(n-2)}$. Let all sections be grids, and in a certain grid there are ordinary senser nodes and heterogeneous sensor nodes which are denoted by IDs. All ordinary sensor nodes are denoted in the section $\mathrm{OM}_{00} \mathrm{M}_{10}$ as $1,2, \mathrm{~L}, \alpha$, next, all heterogeneous sensor nodes are denoted in the section $\mathrm{OM}_{00} \mathrm{M}_{10}$ as $\alpha+1, \alpha+2, \mathrm{~L}, \alpha+\beta$. In the same way, all ordinary sensor nodes are denoted in the section $\mathrm{OM}_{10} \mathrm{M}_{20}$ as $\alpha+\beta+1, \alpha+\beta+2, \mathrm{~L}, 2 \alpha+\beta$, next, all heterogeneous sensor nodes are denoted in the section $\mathrm{OM}_{10} \mathrm{M}_{20}$ as $2 \alpha+\beta+1,2 \alpha+\beta+2, \mathrm{~L}, 2 \alpha+2 \beta$. At last, all ordinary sensor nodes are denoted

in the section $\mathrm{M}_{0(n-2)} \mathrm{M}_{0(n-1)} \mathrm{M}_{7(n-1)} \mathrm{M}_{7(n-2)} \quad$ as $\quad\left(8 n^{2}-2 n+1\right)(\alpha+\beta)+1,\left(8 n^{2}-2 n+1\right)(\alpha+\beta)+2, \mathrm{~L}$, $\left(8 n^{2}-2 n+1\right)(\alpha+\beta)+(2 n-1) \alpha$, next, all heterogeneous sensor nodes are denoted in the section $\mathrm{M}_{0(n-2)} \mathrm{M}_{0(n-1)} \mathrm{M}_{7(n-1)} \mathrm{M}_{7(n-2)} \quad$ as $\quad\left(8 n^{2}-2 n+1\right)(\alpha+\beta)+(2 n-1) \alpha+1,\left(8 n^{2}-2 n+1\right)(\alpha+\beta)+$ $(2 n-1) \alpha+2, \mathrm{~L}, 8 n^{2}(\alpha+\beta)$.

\section{Key systems based identity}

\section{Abilinear map}

We make an assumption that $G_{1}$ is a cyclic additive group and $G_{2}$ is acyclic multiplicative group of the same primer order $q$. Suppose that $\hat{e}: G_{1} \times G_{1} \rightarrow G_{2}$ is a bilinear map. Then,

(1) $\forall P, Q, R, S \in G_{1}, \hat{e}(P+Q, R+S)=\hat{e}(P, R) \hat{e}(P, S) \hat{e}(Q, R) \hat{e}(Q, S)$. $\forall c, d \in Z_{q}, \hat{e}(c P, d Q)=\hat{e}(c P, Q)^{d}=\hat{e}(P, d Q)^{c}=\hat{e}(P, Q)^{c d}$.

(2) $\hat{e}(P, P)$ is the generating element of $G_{2}$ for $P$, the generating element of $G_{1}$.

(3) $\forall P, Q \in G_{1}$, there is an arithmetic to calculate $\hat{e}(P, Q)$. Map $\hat{e}$ is symmetrical. $\forall P, Q \in G_{1}$, $\hat{e}(P, Q)=\hat{e}(Q, P)$.

Define that $p$ and $q$ are two big prime, $E / F_{p}$ is elliptic curve $y^{2}=x^{2}+a x+b$ which is in finite domain $F_{p}, G_{1}$ is additive subgroup order $q$ of points which are on elliptic curve and $G_{2}$ is multiplicative subgroup which is in finite domain $F_{p}$.

\section{The elliptic curve discrete logarithm}

Elliptic curve $E$ is defined in finite domain $F_{p}$ and point $p \in E\left(F_{p}\right)$. Let $<p>$ be cyclic group generated by point $p$. For arbitrary $Q \in<p>$, we get the solution, $l$, of the equation $l P=Q$ which is the elliptic curve discrete logarithm. The difference of the elliptic curve discrete logarithm is the fundament of all cyclic group key schemes. 


\section{Setting up pairwise key based a bilinear map}

\section{the pre-distrbution phase}

System parameters, $p, q, E / F_{p}, G_{1}, G_{2}$, and $\hat{e}$, are generated, and then those parameters are loaded into all those sensors including ordinary sensors and heterogeneous sensors.

\section{Pairwise key establishment}

Assume that those identities of sensors $u$ and $v$ in one same grid are $\left(p_{u}, q_{u}, I D_{u}\right)$ and $\left(p_{v}, q_{v}, I D_{v}\right)$ respectively and assume $p, q, I D_{u}, I D_{v} \in G_{1} . \quad$ it is obvious that $p_{u}=p_{v}=p, q_{u}=q_{v}=q, I D_{u} \neq I D_{v}$. In this case, node $u$ calculates the key $k_{u}\left(I D_{v}\right)$

$$
k_{u}\left(I D_{v}\right)=\hat{e}\left(p+q, I D_{u}+I D_{v}\right)=\hat{e}\left(p, I D_{u}\right) \hat{e}\left(p, I D_{v}\right) \hat{e}\left(q, I D_{u}\right) \hat{e}\left(q, I D_{v}\right)
$$

Similaly, node $v$ calculates the key $k_{v}\left(I D_{u}\right)$

$k_{v}\left(I D_{u}\right)=\hat{e}\left(p+q, I D_{v}+I D_{u}\right)=\hat{e}\left(p, I D_{v}\right) \hat{e}\left(p, I D_{u}\right) \hat{e}\left(q, I D_{v}\right) \hat{e}\left(q, I D_{u}\right)=\hat{e}\left(p, I D_{u}\right) \hat{e}\left(p, I D_{v}\right) \hat{e}\left(q, I D_{u}\right)$ $\hat{e}\left(q, I D_{v}\right)=k_{u}\left(I D_{v}\right)$

So, sensors $u$ and $v$ obtain the same shared key. Generally, two sensors $u$ and $v$ in the same grid can establish shared key $k_{u v}=k_{u}\left(I D_{v}\right)=k_{v}\left(I D_{u}\right)$.

Let $\mathrm{OM}_{00} \mathrm{M}_{10}$ be denoted as $\mathrm{M}_{0(-1)} \mathrm{M}_{00} \mathrm{M}_{10} \mathrm{M}_{1(-1)}, \mathrm{L}, \mathrm{OM}_{60} \mathrm{M}_{70}$ be denoted as $\mathrm{M}_{6(-1)} \mathrm{M}_{60} \mathrm{M}_{70} \mathrm{M}_{7(-1)}, \mathrm{OM}_{70} \mathrm{M}_{00}$ be denoted as $\mathrm{M}_{7(-1)} \mathrm{M}_{70} \mathrm{M}_{00} \mathrm{M}_{0(-1)}$. Without loss of generality, heterogeneous sensors in the same grid but in the same concentric ring, for example, section $\mathrm{M}_{m_{1}\left(n^{\prime}-1\right)} \mathrm{M}_{m_{1} n^{\prime}} \mathrm{M}_{\left(m_{1}+1\right) n^{\prime}} \mathrm{M}_{\left(m_{1}+1\right)\left(n^{\prime}-1\right)} \quad\left(0 \leq m_{1} \leq 7 \quad, \quad 0 \leq n^{\prime} \leq n-1 \quad\right)$ and section $\mathrm{M}_{m_{2}\left(n^{\prime}-1\right)} \mathrm{M}_{m_{2} n^{\prime}} \mathrm{M}_{\left(m_{2}+1\right) n^{\prime}} \mathrm{M}_{\left(m_{2}+1\right)\left(n^{\prime}-1\right)}\left(0 \leq m_{2} \leq 7,0 \leq n^{\prime} \leq n-1\right)$, can establish their shared key. If $m_{2}-m_{1}=1$, those sections are close and $\bmod \left(2 n^{\prime}+1\right)(\alpha+\beta)$ is utilized, where $0 \leq n^{\prime} \leq n-1$. A heterogeneous node $\eta_{h r}$ in section $\mathrm{M}_{m_{1}\left(n^{\prime}-1\right)} \mathrm{M}_{m_{1} n^{n}} \mathrm{M}_{\left(m_{1}+1\right) n^{\prime}} \mathrm{M}_{\left(m_{1}+1\right)\left(n^{\prime}-1\right)}$ can find out the unique heterogeneous sensor node $\mu_{h r}$ in the section $\mathrm{M}_{m_{2}\left(n^{\prime}-1\right)} \mathrm{M}_{m_{2} n^{\prime}} \mathrm{M}_{\left(m_{2}+1\right) n^{\prime}} \mathrm{M}_{\left(m_{2}+1\right)\left(n^{\prime}-1\right)}$ in which $I D_{v_{h r}}-I D_{u_{h r}}=\left(2 n^{\prime}+1\right)(\alpha+\beta)$. The identities of nodes $\eta_{h r}$ and $\mu_{h r}$ are $\left(p_{\eta_{h r}}, q_{\eta_{h r}}, I D_{\eta_{h r}}\right)$ and $\left(p_{\mu_{h r}}, q_{\mu_{h a}}, I D_{\mu_{h r}}\right)$ respectively. It is clear that $p_{\eta_{h r}}=p_{\mu_{h r}}, q_{\eta_{h r}} \neq q_{\mu_{h r}}, I D_{u_{h r}}=I D_{v_{h r}} \bmod \left[\left(2 n^{\prime}+1\right)(\alpha+\beta)\right]$. Here, let $p_{\eta_{h r}}=p_{\mu_{h r}}=P, I D_{u_{h r}}=I D_{v_{h r}} \bmod \left[\left(2 n^{\prime}+1\right)(\alpha+\beta)\right]=Q, \quad q_{\eta_{h r}}=R, q_{\mu_{h r}}=S$, node $\eta_{h r}$ calculates the key $k_{\eta_{h r}}\left(I D_{\mu_{h r}}\right)$

$$
k_{\eta_{h r}}\left(I D_{\mu_{h r}}\right)=\hat{e}(P+Q, R+S)=\hat{e}(P, R) \hat{e}(P, S) \hat{e}(Q, R) \hat{e}(Q, S)
$$

Similaly, node $\mu_{h r}$ calculates the key $k_{\mu_{h r}}\left(I D_{\eta_{h r}}\right)$

$$
k_{\eta_{h r}}\left(I D_{\mu_{h r}}\right)=\hat{e}(P+Q, S+R)=\hat{e}(P, R) \hat{e}(P, S) \hat{e}(Q, R) \hat{e}(Q, S) k_{\eta_{h r}}\left(I D_{\mu_{h r}}\right)
$$

Therefore, a heterogeneous node $\eta_{h r}$ and a heterogeneous sensor node $\mu_{h r}$ obtain the same shared key, and heterogeneous sensors not in the same grid but in the same concentric ring can obtain their shared keys.

By the same way, heterogeneous sensors not in the same grid but in the same sector can obtain their shared keys.

\section{The scheme performance analysis}

\section{The security analysis}

Two sensor nodes, $u$ and $v$, in one certain grid share common key $k_{u}\left(I D_{v}\right)=k_{v}\left(I D_{u}\right)$. Those keys are obtained through using identities of sensors $u$ and $v$ and the elliptic curve discrete logarithm. Those identities in different grids are different. Therefor, if some nodes are captured, they can not threat other sensors in the grid. This scheme has good security. Moreover, even in the same grid, other 
nodes are not compromised by the captured nodes because it is diffrent to obtain the solution for the elliptic curve discrete logarithm.

\section{The connectivity analysis}

All those sensors including ordinary snesors and heterogeneous sensors in the same grid can set up their keys and can communicate safely. Moreover, all those heterogeneous sensors in the same concentric ring can establish their keys and all those heterogeneous sensors in the same sector can establish their keys too. So, all heterogeneous sensors can set up their keys directly or through employing other heterogeneous sensors indirectly. It is obvious that all ordinary sensors can establish their keys in the same grid directly or establish their keys not in the same grid indirectly through utilizing those heterogeneous sensors. Therefore, this scheme has good connectivity.

\section{Conclusion}

The strategy is obtained through combining a bilinear map and the elliptic curve discrete logarithm after dividing sensing area into many different grids. The scheme has high security and good network connectivity.

\section{Acknowledgements}

This work was supported by the Project of Shandong Province Higher Educational Science and Technology Program, and the project number is J13LN05.

\section{References}

[1] J. Ford. "Telecommunications with MEMS devices: an overview", in: Proceedings of the 14th Annual Meeting of the IEEE Lasers and Electro-Optics Society, vol. 2,no.12, November 2001, pp.415-416, (2001).

[2] Yuquan Zhang. A Secure Scheme for Heterogeneous Wireless Sensor Networks[J], Journal of Theoretical and Applied Information Technology, 2013(48):570-576.

[3] L. Eschenauer and V. D. Gligor, “A key-management scheme for distributed sensor networks," in Proc. CCS'02: 9th ACM Conference on Computer and Communications Security. New York: ACM Press, Nov. 2002, pp.41-47, (2002).

[4] H. Chan, A. Perrig, D. Song. "Random key predistribution schemes for sensor networks", Proceedings of the IEEE Syrup. On Research in Security and Privacy, Berkeley, CA, USA, May 11-14 2003, pp.197-213, (2003).

[5] D. Liu, P. Ning. "Establishing pairwise keys in distributed sensor networks", In: Proceedings of 10th ACM conference on computer and communications security (CCS03). Washington, DC: ACM Press, pp.41-47, (2003). 